\title{
Magnetorheological elastomer stress relaxation behaviour during compression: experiment and modelling
}

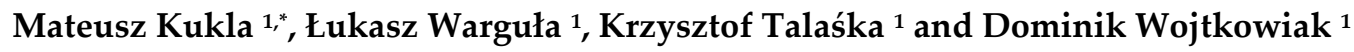 \\ 1 Institute of Machine Design, Faculty of Mechanical Engineering, Poznan University of Technology, \\ Piotrowo 3, PL-60965 Poznan, Poland; lukasz.wargula@put.poznan.pl (Ł.W.); \\ krzysztof.talaska@put.poznan.pl (K.T.); dominik.wojtkowiak@put.poznan.pl (D.W.) \\ * Correspondence: mateusz.kukla@put.poznan.pl (M.K.) +48 612244514
}

\begin{abstract}
Materials characterised by magnetorheological properties are non-classic engineering materials. A significant increase in the interest of scientific community in materials from this group can be observed over the recent several years. The results of research presented in this article are oriented on the examination of said materials' mechanical properties. In order to do so, stress relaxation tests were conducted on cylindrical samples of magnetorheological elastomers loaded with compressive stress for various values of magnetic induction $\left(B_{1}=0 \mathrm{mT}, B_{2}=32 \mathrm{mT}, B_{3}=48\right.$ $\mathrm{mT}$, and $\left.B_{4}=64 \mathrm{mT}\right)$ and temperature $\left(T_{1}=25^{\circ} \mathrm{C}, T_{2}=30^{\circ} \mathrm{C}\right.$, and $\left.T_{3}=40^{\circ} \mathrm{C}\right)$. The results of these tests indicate that the stiffness of examined samples increases along with the increase of magnetic field induction and decreases along with the increase of temperature. On this basis, it has been determined that: the biggest stress amplitude change caused by the influence of magnetic field was $\sigma_{0}^{\Delta B}=12.7 \%$ and the biggest stress amplitude change caused by the influence of temperature was $\sigma_{0}^{\Delta T}=11.3 \%$. As a result of applying a mathematical model, it has been indicated that the stress relaxation in the examined magnetorheological elastomer for the adopted time range $(t=3600 \mathrm{~s})$ has a hyperbolic decline nature. The collected test results point to examined materials being characterised by extensive rheological properties, which leads to a conclusion that it is necessary to conduct further tests in this scope.
\end{abstract}

Keywords: mechanical properties of advanced materials; magneto-rheological (MR); elastomer; stress relaxation; mathematical model

\section{Introduction}

Magnetorheological elastomers (MRE) are a group of composite materials, the mechanical properties of which change under the influence of applied magnetic field [1]. This change occurs in a time range counting up to several milliseconds and is fully reversible $[2,3]$. They combine materials with various properties: elastic polymers and metals with good magnetic properties. Magnetorheological elastomers are often referred to as solid state magnetorheological fluids [4]. However, certain differences exist between those two material groups. The most important difference is that magnetic particles in elastomer composites have a very limited ability to move in the matrix [5]. The change of their position is in practice possible only through elastic deformation of the matrix solid material. Due to differences in structure, magnetorheological elastomers operate in a load range other than fluids, i.e. below the yield point [6]. This two types of materials are rather complementary than competitive due to nature of their work.

Due to the unique properties of magnetorheological elastomers, many researchers focus their attention on the behaviour of elastomers in a magnetic field. The most often conducted tests concern the identification of material properties spectrum related to the composite being loaded with shear stress [7]. However, polymer matrix-based composites from this group can also transfer different types of loads. Therefore, it is possible to indicate applications not only in torsion [8-10], but also in 
compression mode [11]. Thus, it is quite clear that the test of composite materials' mechanical properties, for example, during compression is also extremely important [12-14].

Designing devices utilising magnetorheological elastomers in practical applications requires the repeatability of their behaviour and upkeep of set settings resulting from their properties. A longterm and fault-free operation of devices using discussed materials requires a thorough recognition of their properties both in relation to time and temperature. Elastomer materials are characterised by the decline of their mechanical properties with the passage of time. Such process is known as aging [15]. Additionally, the mechanical properties of polymers in general are strongly depend on temperature [16, 17]. The tests of Wan et al. from 2018 show that the glass transition of magnetorheological samples manufactured on the basis of silicone rubber at uniaxial compression occurs at the temperature of approximately $50^{\circ} \mathrm{C}$. The value of storage modulus points to two different trends occurring along with temperature changes: at first it rapidly declines and then it slightly increases or maintains a constant value as the temperature increases [18]. Systems that will use MRE can heat up as a result of heat radiating from co-operating devices, e.g. drive units [19], gearboxes [20], or working mechanisms [21]. Energy dissipation (resulting from regular operation) also increases the temperature of polymer materials due to internal friction occurrence [22]. Moreover, they are characterised by complex rheological properties that characterise, among other things, creep and stress relaxation phenomena [23]. For that reason, it is justified to conduct research aimed at examination of aforementioned properties. Research issues related to the stress relaxation can also be found in works [24-26].

The purpose of presented research was to determine the impact of magnetic field induction $B$, temperature $T$, and time $t$ on mechanical properties of magnetorheological elastomers subjected to the uniaxial compressive stress. The analysis of available literature regarding magnetorheological elastomers and polymers served as a basis for hypothesis that the increase of stiffness in examined samples will depend on the increase of parameters characterising the magnetic field and will be inversely dependent on the increase of temperature.

\section{Materials and Methods}

\subsection{Sample preparation}

As part of the research, testes was undertaken on the stress relaxation of the studied magnetorheological elastomers. The test consisted of loading samples to a set deformation value and then maintaining the said deformation for a set amount of time. Elastomer materials (and thus also their composites) are characterised by viscoelastic properties and for that reason it is expected that stress in a sample loaded in such way will gradually decrease. In other words, the stress relaxation phenomenon will occur. Samples were made as a result of combining the Sika-brand Biresin U1404 elastomer and carbonyl iron powder with particle diameter between 6 and $9 \mu \mathrm{m}$. The carbonyl iron powders are commonly used magnetic particles in process of MRE preparation [27]. Specific components were combined through mechanical mixing followed by degassing and placing in a mould designed specifically for this purpose. The diagram of mould is shown in Figure 1a. The aluminium alloy mould 4 is located between two steel covers 1 . Inside the mould are located channels that give the magnetorheological elastomer 3 the required geometrical properties. The mould is fixed to the cover with the help of bolts 5 . The tight closure of mould by covers 1 is ensured by properly tensioned aluminium alloy bolts 2 . During sample curing, the mould was subjected to magnetic field along their axles. The appropriate selection of applied materials makes the magnetic flux closes through the sample during its manufacturing. This occurs because the applied aluminium alloy and air have significantly lower magnetic permeability than the mould covers and the magnetorheological composite itself. The created samples had the shape of cylinders with diameter $d=20 \mathrm{~mm}$ and height $h=20 \mathrm{~mm}$. The curing of samples was carried out in room temperature and in the presence of magnetic field with magnetic induction $B=300 \mathrm{mT}$. The samples were manufactured in such an amount that each iteration could be conducted on a new sample - this 
eliminated the necessity of taking the history of sample loading into account. The microscope photos of examined samples' cross-sections are shown in Figure $1 \mathrm{~b}$ - iron particles are presented as brighter fields.
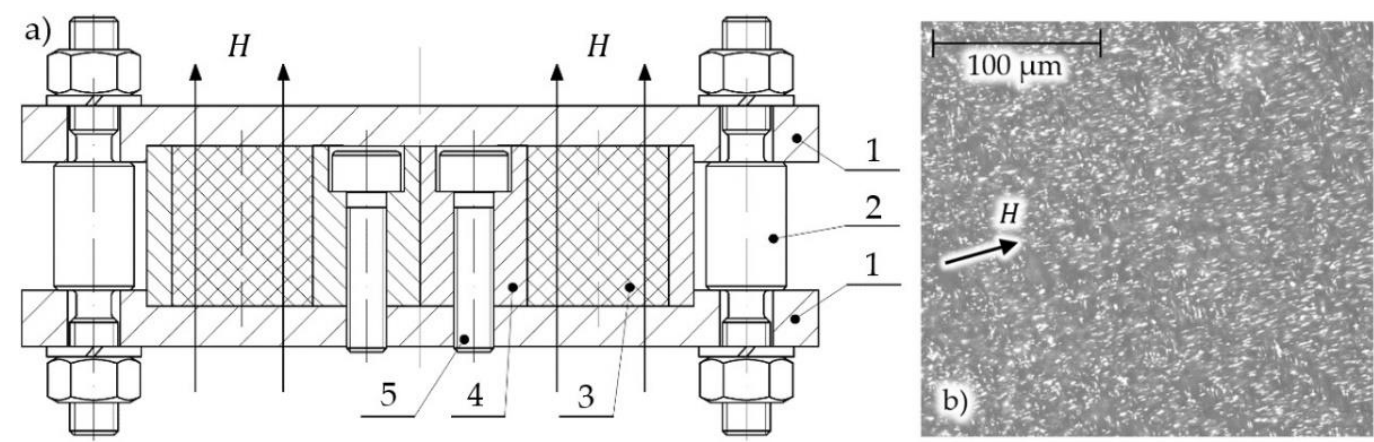

Figure 1. (a) MRE sample manufacturing mould diagram; 1 - mould covers, 2 - cover fixing elements, 3 magnetorheological elastomer, 4 - mould, 5 - mould fixing elements; (b) microscope photo of internal sample structure

\subsection{Experiment detalis}

The tests were carried out on the MTS Insight universal testing machine. The measurement of force value was conducted using HBM-brand C9C $1 \mathrm{kN}$ sensor. An HBM-brand WA $20 \mathrm{~mm}$ linear position sensor was used to determine the displacement value. The whole system co-operated with Spider 8 measurement system connected to a PC. In addition, the system was equipped in specially designed, original test stand, the purpose of which was to create a magnetic field with set parameters, in order to conduct further experiments. The system's diagram is shown in Figure 2. Housing 2, as well as covers, was made from a special iron alloy characterised by good magnetic properties. After machining, the housing elements were subjected to annealing in order to acquire uniform properties in their whole cross-section. Inside the housing is located an induction coil 4 along with magnetorheological elastomer sample 3. The whole system is fixed to a base made from non-magnetic austenitic steel. This ensures the closure of magnetic flux through the examined sample.

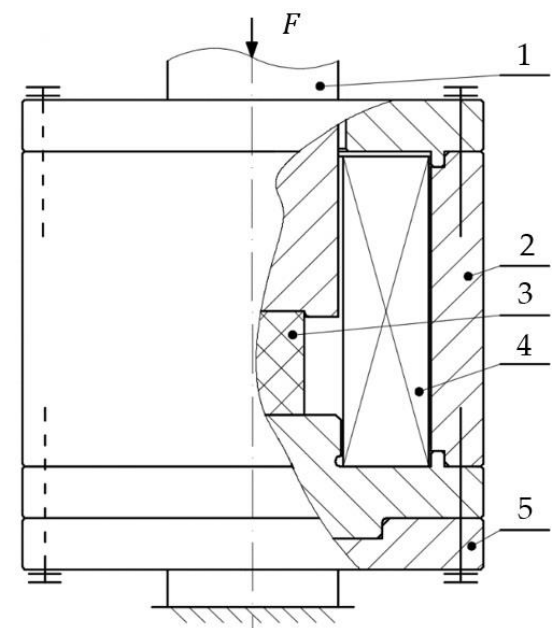

Figure 2. Study position diagram; 1 - element transferring compressive force, 2 - housing, 3 - examined sample, 4 - coil, 5 - base 
The test stand was placed in a climate chamber dedicated for the utilised universal testing machine, which allowed to control and measure the temperature inside its working space. In addition, the control of temperature value during experiments was carried out using Seek Thermalbrand RevealPRO FF RQEAAX thermal vision camera. The samples were compressed to a set deformation amplitude $\varepsilon=20 \%$ with speed $v=0.8 \mathrm{~m} / \mathrm{s}$. For conditions set in such a way, a sample relaxation was conducted in time range equal to $t=3600 \mathrm{~s}$, in which the variable parameters were constituted by magnetic induction values $B_{1}=0 \mathrm{mT}, B_{2}=32 \mathrm{mT}, B_{3}=48 \mathrm{mT}$, and $B_{4}=64 \mathrm{mT}$ and temperatures $T_{1}=25^{\circ} \mathrm{C}, T_{2}=30^{\circ} \mathrm{C}$, and $T_{3}=40^{\circ} \mathrm{C}$. The measurement system diagram is shown in Figure $3 \mathrm{a}$ while its view is shown in Figure $3 \mathrm{~b}$.
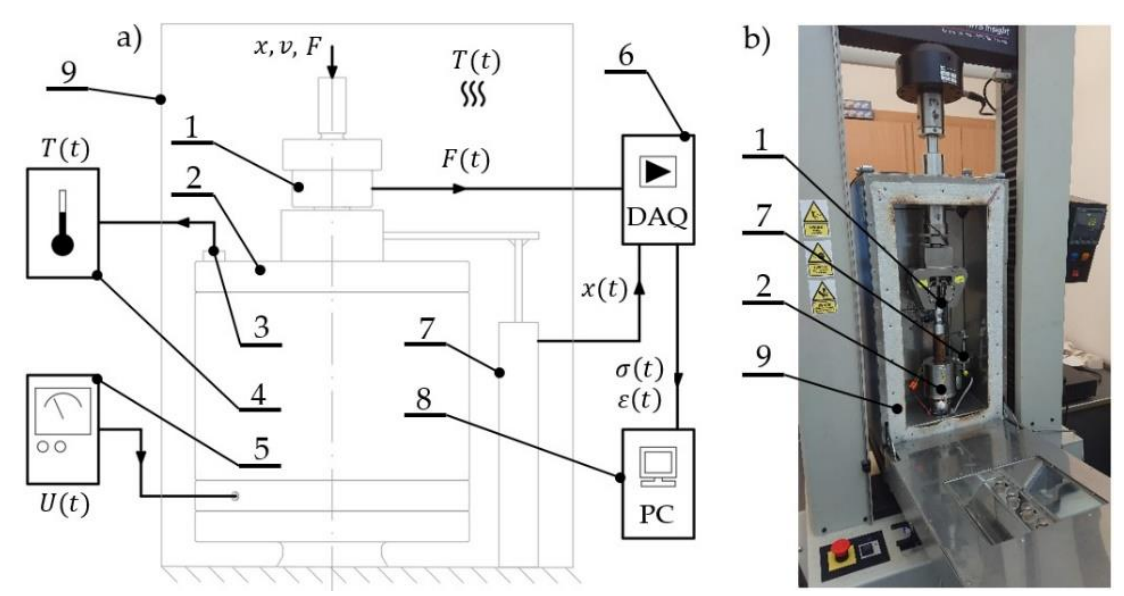

Figure 3. Measurement system: (a) diagram, (b) view: 1 - force sensor, 2 - measurement position, 3 - temperature sensor, 4 - temperature converter, 5 - laboratory power supply unit, 6 - measurement amplifier, 7 - displacement sensor, 8 - computer, 9 - climate chamber

\section{Results and Discussion}

\subsection{Experimental research}

Figure 4 shows the initial range of sample load relaxation test results for various magnetic induction $B$ values. Figure 5 shows the initial range of sample load relaxation test results for various magnetic induction $t$ values while Figure 6 shows the same results for the whole analysed time range $T$.

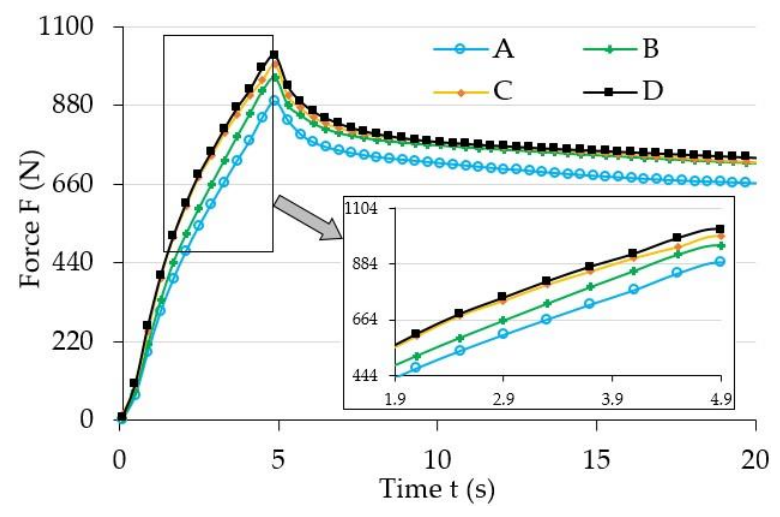

Figure 4. Selected test results for various magnetic field induction $B$ values for temperature and value $T=25^{\circ} \mathrm{C}$; A - $0 \mathrm{mT}, \mathrm{B}-32 \mathrm{mT}, \mathrm{C}-48 \mathrm{mT}, \mathrm{D}-64 \mathrm{mT}$ 


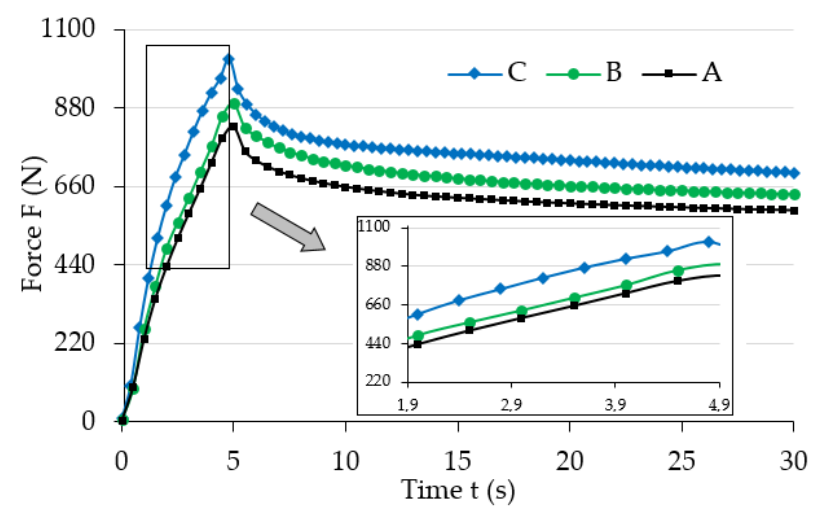

Figure 5. Selected test results for various temperature $T$ values for magnetic induction with value $B=0 \mathrm{mT}$; temperature value: $\mathrm{A}-25^{\circ} \mathrm{C}, \mathrm{B}-30^{\circ} \mathrm{C}, \mathrm{C}-40^{\circ} \mathrm{C}$

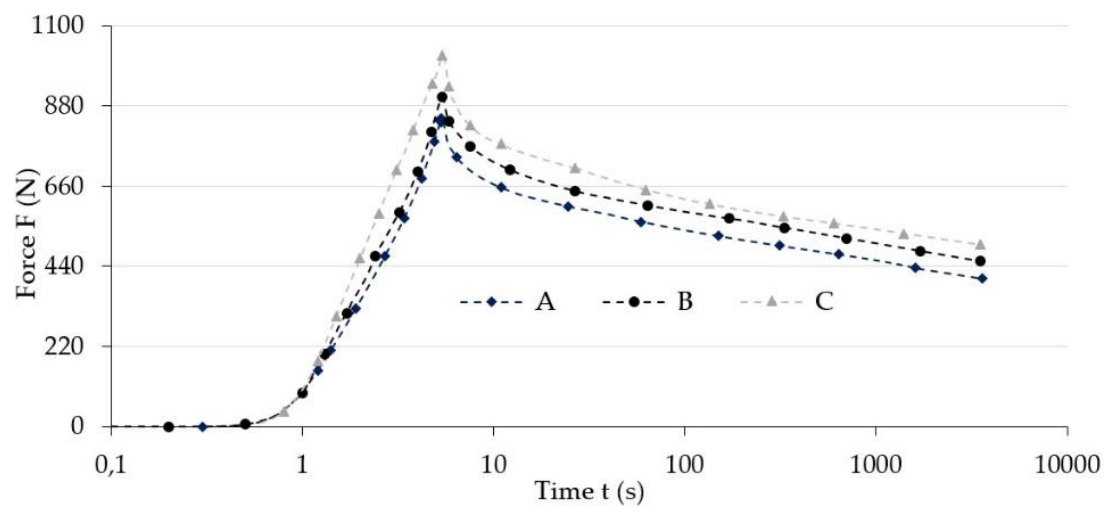

Figure 6. Selected test results for various temperature $T$ values for magnetic induction with value $B=0 \mathrm{mT}$ (in the logarithmic scale of time - for the whole duration of a single experiment); temperature value: $\mathrm{A}-25^{\circ} \mathrm{C}, \mathrm{B}-$ $30^{\circ} \mathrm{C}, \mathrm{C}-40^{\circ} \mathrm{C}$

The analysis of results (Figure 4) allows to notice the increase of maximum force (strain) value for examined samples that occurred along with the increase of magnetic field induction $B$. It also has an influence on the progress of stress relaxation process in the examined samples. The collected results suggest that the increase of magnetic field parameters caused the increase (extension) of load relaxation time. Such phenomena can be explained by mutual influence of magnetic field and ferromagnetic particles. During manufacturing they show a tendency to group in columns according to the direction of applied magnetic field [28] due to magnetisation. In addition, ferromagnetic particles aim to properly orient their easy magnetisation directions in space, that is, in parallel to the line of magnetic flux. Their arrangement is the result of system's attempt to reach the state of minimum energy. In this position they will be fixed after the curing completion of the polymer matrix. Later deformation of such material requires greater amount of energy, i.e. performing additional work (applying greater force) when compared to the deformation of magnetorheological elastomer not subjected to the magnetic field. This energy is used to relocate ferromagnetic particles from positions with minimum energy. The amount of required work to be supplied depends on the intensity of applied magnetic field [29]. Thus, an apparent increase of mechanical properties, for example, the stiffness module, which are the function of magnetic field induction, will be observed when analysing the properties of MRE samples in a macro-scale. The value of stiffness module has a direct impact on the progress of discussed process in relation to both the maximum force (load) value and stress relaxation time.

The analysis of results (Figures 5 and 6) allows to notice the decline of maximum force (load) value for examined samples that occurs along with the increase of temperature $T$. The stress relaxation phenomenon depends on temperature because the increase of temperature causes a 
proportional reduction of internal friction, which in consequence shortens the stress relaxation time. This phenomenon also depends on the polymer's structure and type of network and bonds created in the vulcanizate. This is caused by the fact that the structure of elastomers has a macro-particle structure. The molecular chains that comprise the composite matrix have the ability to move in relation to each other. Therefore, materials from this group are characterised by the occurrence of mechanical hysteresis, which is one of few energy dissipation ability indicator. The increase of temperature results in the increased mobility of molecular chains and, as a consequence, reduction of load value necessary to deform samples. Thus, a decline of mechanical properties, for example the stiffness module, which are the function of temperature, will be observed when analysing the properties of MRE samples in a macro-scale.

The properties of magnetorheological elastomer as a composite material strongly depend on its manufacturing method and materials used for this purpose [2]. Quite often, various non-magnetic additives for polymer matrix material are used to change the MRE mechanical properties while maintaining the fixed content of magnetic phase [30]. As a material group, elastomers are characterised by strongly developed rheological properties. For this reason, the influence of amplitude and deformation velocity on the MRE properties also cannot be omitted in general. Those two values in the discussed experiment were fixed and therefore their influence on the recorded results was not determined. This was taken into account when determining the maximum load value $\sigma_{0}$ as a function of temperature $T$ and magnetic field induction $B$, which has been shown in Figure 7. This physical quantity carries the information about stiffness of examined composite and can be used as an indicator of upcoming changes in mechanical properties. Depending on magnetic induction $\sigma_{0}^{\Delta B}$, the values of relative stress amplitude increases have been determined according to dependence (1):

$$
\sigma_{0}^{\Delta B}=\frac{\sigma_{0}^{B \max }-\sigma_{0}^{B \min }}{\sigma_{0}^{B \min }} \cdot 100 \%,
$$

where: $\sigma_{0}^{\Delta B}$ is a relative stress amplitude increase depending on magnetic induction, $\sigma_{0}^{B m a x}$ is the greatest strain amplitude value for the greatest magnetic induction value for a given temperature and $\sigma_{0}^{B m i n}$ is the greatest strain amplitude value for the greatest magnetic induction value for a given temperature. The values of relative stress amplitude increases depending on temperature $\sigma_{0}^{\Delta T}$, have been determined in a similar way. The summary of calculated results are presented in Table 1. As it can be seen, the biggest stress amplitude change caused by the influence of magnetic field was $\sigma_{0}^{\Delta B}$ $=12.7 \%$ and the biggest stress amplitude change caused by the influence of temperature was $\sigma_{0}^{\Delta T}=$ $11.3 \%$.

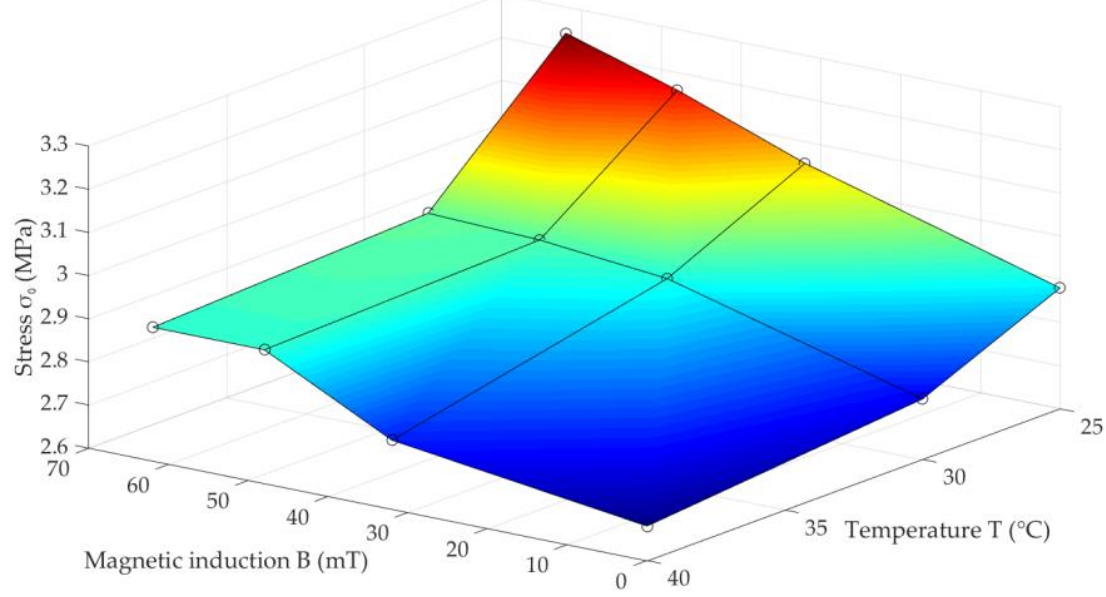

Figure 7. Stress amplitude $\sigma_{0}$ dependency on magnetic field induction $B$ and temperature $T$ for the examined MRE samples 


\begin{tabular}{|c|c|c|c|c|c|c|}
\hline & & \multicolumn{4}{|c|}{ Magnetic induction $B(\mathrm{mT})$} & \multirow{3}{*}{$\sigma_{0}^{\Delta B}(\%)$} \\
\hline & & 0 & 32 & 48 & 64 & \\
\hline & & \multicolumn{4}{|c|}{ Stress amplitude $\sigma_{0}(\mathrm{MPa})$} & \\
\hline \multirow{3}{*}{ Temperature $T\left({ }^{\circ} \mathrm{C}\right)$} & 25 & 2.88 & 3.05 & 3.16 & 3.24 & 12.7 \\
\hline & 30 & 2.74 & 2.90 & 2.93 & 2.94 & 7.2 \\
\hline & 40 & 2.68 & 2.76 & 2.91 & 2.92 & 8.8 \\
\hline$\sigma_{0}^{\Delta T}(\%)$ & & 7.5 & 10.3 & 8.6 & 11.3 & \\
\hline
\end{tabular}

\subsection{Mathematical model}

The initial attempts focusing on the application of standard rheological model in the form of an equation (2):

$$
\sigma(t)=E_{2} \varepsilon_{0}+E_{1} \varepsilon_{0} \mathrm{e}^{-\frac{E_{1}}{\eta} t},
$$

where: $E_{1}$ and $E_{2}$ are elasticity moduli, $\varepsilon_{0}$ is strain amplitude, $\eta$ is viscosity, and $t$ is time, gave unsatisfactory results for the mathematical description of examined composites' properties. Therefore, a decision to apply model presented in [31] was made. Its Authors have led out mathematical relationships intended for the description of stress relaxation phenomenon in the form of an equation (3):

$$
\frac{1}{\sigma^{m-1}(t)}=\frac{1}{\sigma_{0}^{m-1}}\left[1+(m-1) \frac{E}{\eta} t\right]
$$

where: $m$ is steady-state creep exponent and $\sigma_{0}$ is stress amplitude. After simple transformations, the equation (3) can be shown in the form of a dependency (4):

$$
\sigma(t)=\sigma_{0}\left[1+(m-1) \frac{E}{\eta} t\right]^{-\frac{1}{m-1}}
$$

In order to simplify the notation of equation (4), functions (5) can be substituted:

$$
a=\frac{\eta}{E} \quad \text { and } \quad b=(m-1), \quad \text { as well as } \quad \sigma_{0}=q,
$$

where: $a$ is load extenuating rate of the material and $b$ is a function of $m$ (steady-state creep exponent). As a result, the final form of equation acquired is given as (6):

$$
\sigma(t)=q\left[1+\frac{b}{a} t\right]^{-\frac{1}{b}}
$$

The analysis of equations (2) and (6) allows to draw a conclusion that both equations allow to model a decreasing tendency. The value of stress $\sigma(t)$ declines along with the increase of time $t$ in each one of them. However, from the mathematical point of view the equation (2) models an exponential decline while dependency (6) has a hyperbolic decline nature. For this reason, it seems that it is significantly better to fit the data recorded during experiments.

This observation served as a basis for conducting numeric calculations aimed at determining a pair of equation (6) $a$ and $b$ parameters in a way that the acquired curve allows to fit the collected measurement data as accurately as possible. The procedure used during this activity was based on the least squares method. Parameter $q$ corresponds to the stress amplitude $\sigma_{0}$ and its value was read directly from experimental data. The summary of acquired characteristics in magnetic induction function is shown in Figure 8. For illustrative purposes, a characteristics that shows the dependency of factor $a$ on temperature $T$ was additionally included in Figure 9. The summary of results acquired: due to conducting an experiment, as a result of applying model described with 
equation (2) and in accordance with model (6) are shown in Figure 10. As it can be seen, the latter is characterised by a significantly better quality of rendering set values.
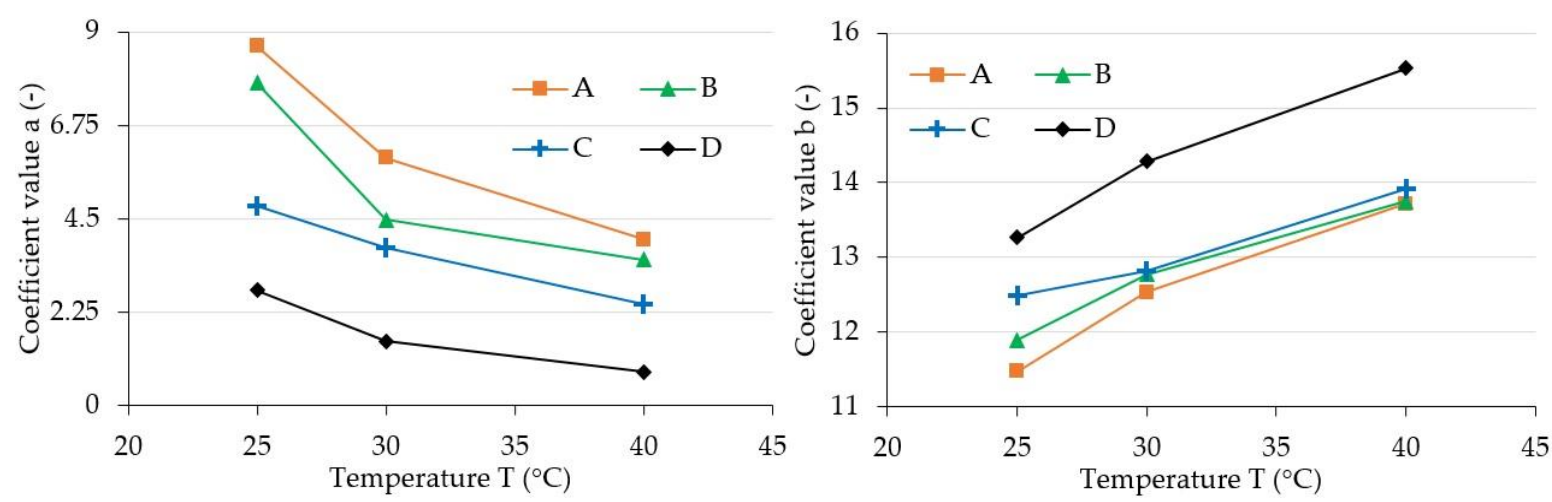

Figure 8. Set values of $a$ and $b$ coefficients for various magnetic induction values; A - $64 \mathrm{mT}, \mathrm{B}-48 \mathrm{mT}, \mathrm{C}-32$ $\mathrm{mT}, \mathrm{D}-0 \mathrm{mT}$
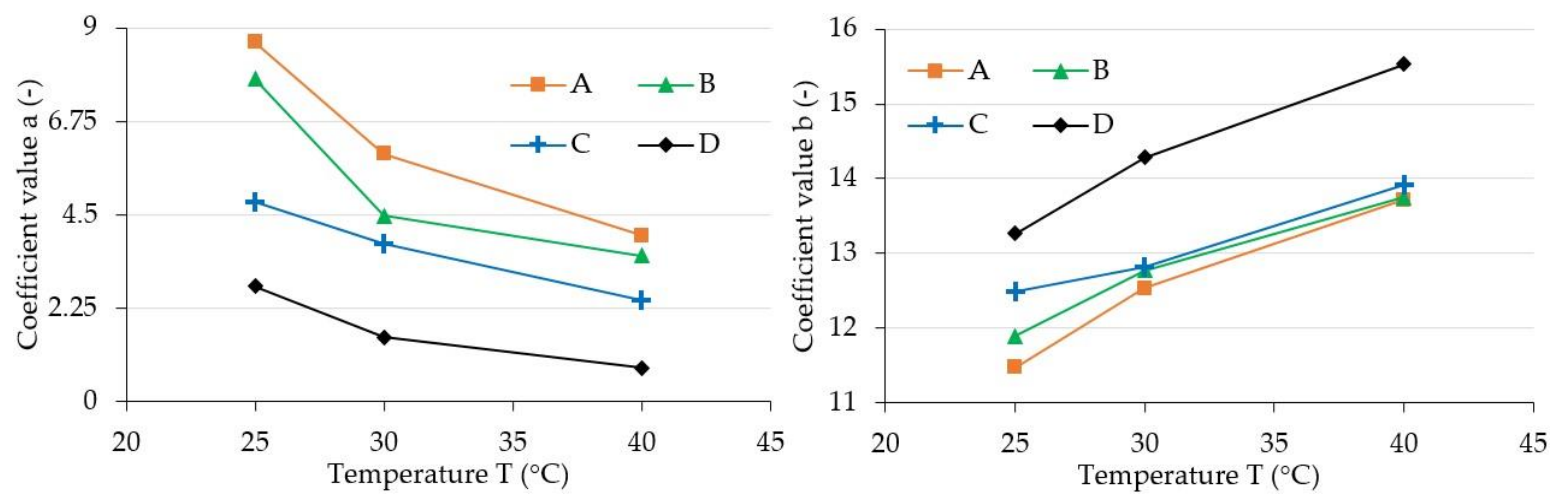

Figure 9. Set values of coefficients; a) $q$ for various magnetic induction values: A - $64 \mathrm{mT}, \mathrm{B}-48 \mathrm{mT}, \mathrm{C}-32 \mathrm{mT}$, $\mathrm{D}-0 \mathrm{mT}$; b) for various temperature values: $\mathrm{E}-25^{\circ} \mathrm{C}, \mathrm{F}-30^{\circ} \mathrm{C}, \mathrm{G}-40^{\circ} \mathrm{C}$

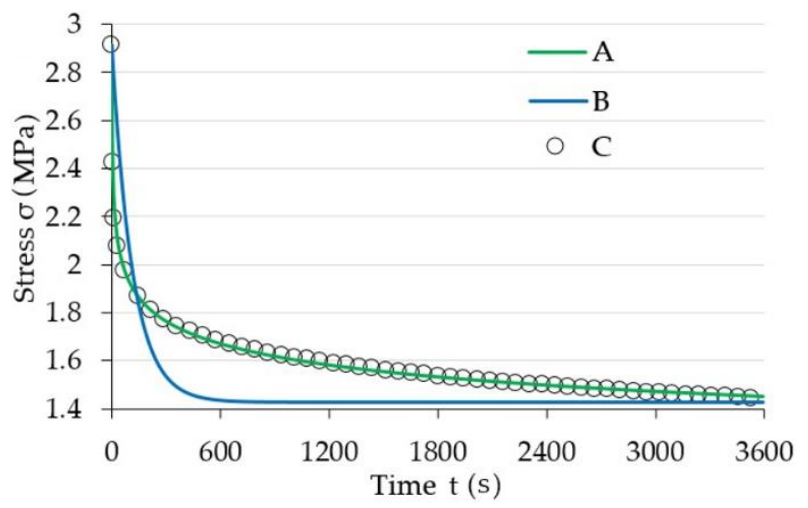

Figure 10. Summary of modelling results; A - rheological model acc. to [31], B - standard rheological model, C - measurement data

According to Authors of model (2) [31], the value of parameter $a$ expresses the load extenuating rate of the material. The value $m$, which defines parameter $b$, is the steady-state creep exponent. Parameter $q$ is straightforward stress amplitude $\left(\sigma_{0}\right)$, which results from the greatest force value recorded in the experiment. The analysis of included figures allows to draw a conclusion that the parameter $a$ and $q$ values decline with the temperature increase while the value of parameter $b$ 
increases. This is the effect of previously described change of composite matrix material property, which is the result of internal friction change. The impact of magnetic field expressed through magnetic induction $B$ results in the increase of parameter $b$ and $q$ values. This results from the internal structure of magnetorheological elastomer, thus from the mutual influence of magnetic field and ferromagnetic particles of which the said composite consists.

The tests of magnetorheological elastomer properties in the range between $25^{\circ} \mathrm{C}$ and $40^{\circ} \mathrm{C}$ allow to draw conclusions similar to those drawn during tests of these materials by the Wan et al. team in 2018 that elastomers in this temperature range are characterised by a significant loss of mechanical properties along with temperature increase [18].

\section{Conclusions}

The results of both: own tests and analysis of available literature allow to declare that magnetorheological elastomers are materials with exceptionally complex properties. The properties of manufactured composite depend on numerous factors. In first order, those are: applied materials of components and production methods. The further change of properties is related to the influence caused on ferromagnetic particles included in the composite matrix material by magnetic field. The scale of observed changes also depends on the applied load state as polymer materials can withstand various types of stress, such as: compression, shearing, or bending, as well as any combinations of those. Test results also point to complex rheological properties of magnetorheological elastomers. As a result, the load relaxation and creeping phenomena also carry some significance. Therefore, the important role in the analysis of materials from this group is attributed not only by load and deformation values, but also by the dynamics of their changes in time. The impact of temperature is also non-negligible because it has a crucial meaning for the properties of composite matrix material. In general, the MRE loading history should also be taken into account because the composite matrix material and ferromagnetic material contained in it are characterised by intermolecular interactions. The issue is additionally complicated by the overlap or mutual influence of several physical phenomena. For example, the time, in which the deformation can develop, is proportionally reduced along with the increase of strain velocity. Thus, in the context of stress relaxation time for certain value ranges, temperature reduction results in changes with similar nature as increasing strain velocity and vice versa. Increasing the strain velocity causes the increase of force necessary to deform the elastomer, which is the result and confirmation of viscoelastic properties of that material. The next example is the mutual relation of mechanical and magnetic interactions. The compressive stress causes the MRE deformation, which results in change of distance between ferromagnetic material particles. Their mutual interaction, caused by the magnetic field, depends, inter alia, on their mutual position (distance between the particles).Therefore, in this context it is possible to show a mutual dependency between two different interactions.

To sum up, it should be concluded that magnetorheological elastomers in general are materials with complex properties. Further tests on the composites from this group, as well as mutual relations between physical interactions related to them, are necessary

Author Contributions: Conceptualization, M.K., Ł.W., K.T. and D.W.; methodology, M.K., Ł.W., K.T. and D.W.; software, M.K., Ł.W.; validation, M.K., Ł.W. and K.T.; formal analysis, M.K., Ł.W.; investigation, M.K., Ł.W., K.T. and D.W.; resources, M.K.; data curation, M.K.; writing-original draft preparation, M.K., Ł.W.; writingreview and editing, M.K., Ł.W., K.T. and D.W.; visualization, M.K., Ł.W., K.T. and D.W.; supervision, M.K.; project administration, M.K.; funding acquisition, M.K., Ł.W., K.T. and D.W. All authors have read and agreed to the published version of the manuscript.

Conflicts of Interest: The authors declare no conflict of interest.

\section{References}


1. Lewandowski, D. Modeling of Magnetorheological Elastomers Using the Elastic-Plastic Model with Kinematic Hardening. Materials 2019, 12, 892.

2. Samal, S.; Škodová, M.; Abate, L.; Blanco, I. Magneto-Rheological Elastomer Composites. A Review. Applied Sciences. 2020, 10, 4899.

3. Li, T., El-Aty, A. A., Cheng, C., Shen, Y., Wu, C. et al. Investigate the Effect of the Magnetic Field on the Mechanical Properties of Silicone Rubber-Based Anisotropic Magnetorheological Elastomer during Curing Process. Journal of Renewable Materials, 2020, 8(11), 1411-1427.

4. Miedzińska, D.; Boczkowska, A.; Zubko, K. Numerical verification of three point bending experiment of magnetorheological elastomer (MRE) in magnetic field. Journal of Physics: Conference Series 2010, 240(1).

5. Bica, I.; Bunoiu, O.M. Magnetorheological Hybrid Elastomers Based on Silicone Rubber and Magnetorheological Suspensions with Graphene Nanoparticles: Effects of the Magnetic Field on the Relative Dielectric Permittivity and Electric Conductivity. International Journal of Molecular Sciences 2019, 20, 4201 .

6. Choi, W.J. Dynamic Analysis of Magnetorheological Elastomer Configured Sandwich Structures, Doctoral dissertation 2009, University of Southampton

7. Liao, G.; Gong, X.; Xuan, S.; Guo, C.; Zong, L. Magnetic-field-induced normal force of magnetorheological elastomer under compression status. Industrial E Engineering Chemistry Research 2012, 51(8), 3322-3328.

8. Hoang, N.; Zhang, N.; Du, H. An adaptive tunable vibration absorber using a new magnetorheological elastomer for vehicular powertrain transient vibration reduction. Smart Materials and Structures 2010, 20(1), 1-11.

9. Hoang, N.; Zhang, N.; Li, W.H.; Du, H. Development of a torsional dynamic absorber using a magnetorheological elastomer for vibration reduction of a powertrain test rig. Journal of Intelligent Material Systems 2013, 24, 2036-2044.

10. Mitsumata, T.; Ohori, S. Magnetic Polyurethane Elastomers With Wide Range Modulation of Elasticity, Polymer Chemistry 2011, 2(5), 1063-1067.

11. Sun, S.S.; Chen, Y.; Yang, J.; Tian, T.F.; Deng, H.X.; Li, W.H.; Du, H.; Alici, G. The development of an adaptive tuned magnetorheological elastomer absorber working in squeeze mode. Smart Materials and Structures 2014, 23(075009),1-8.

12. Bica, I. Electroconductive magnetorheological suspensions: production and physical processes. Journal of industrial and engineering chemistry 2009, 15(2), 233-237.

13. Pavlenko, A.V.; Turik, A.V.; Reznichenko, L.A.; Shilkina, L.A.; Konstantinov, G.M. The magnetodielectric effect in Bi 1/2 La 1/2 MnO 3 cramics. Technical Physics Letters 2013, 39(1), 78-80.

14. Mouritz, A.P.; Gardiner, C.P. Compression properties of fire-damaged polymer sandwich composites. Composites Part A: Applied Science and Manufacturing 2002, 33(5), 609-620.

15. Zhang, W.; Gong, X.L.; Jiang, W.Q.; Fan, Y.C. Investigation of the durability of anisotropic magnetorheological elastomers based on mixed rubber. Smart Materials and Structures 2010, 19(8), 085008.

16. Zhang, W.; Gong, X.; Xuan, S.; Jiang, W. Temperature-dependent mechanical properties and model of magnetorheological elastomers. Industrial \& engineering chemistry research 2011, 50(11), 6704-6712.

17. Yu, M.; Zhao, L.; Fu, J.; Zhu, M. Thermal effects on the laminated magnetorheological elastomer isolator. Smart Materials and Structures 2016, 25(11), 115039.

18. Wan, Y.; Xiong, Y.; Zhang, S. Temperature dependent dynamic mechanical properties of Magnetorheological elastomers: Experiment and modeling. Composite Structures 2018, 202, 768-773.

19. Wargula, Ł.; Waluś, K.J.; Krawiec, P. The problems of measuring the temperature of the small engines (SI) on the example of a drive for non-road mobile machines. In MATEC Web of Conferences, Proceedings of the XXIII Polish-Slovak Scientific Conference on Machine Modelling and Simulations, Rydzyna, Poland, 4-7 September 2019; EDP Sciences: Lez Ili, France, 2019; Volume 254.

20. Krawiec, P.; Różański, L.; Czarnecka-Komorowska, D.; Warguła, Ł. Evaluation of the Thermal Stability and Surface Characteristics of Thermoplastic Polyurethane V-Belt. Materials 2020, 13(7), 1502.

21. Yashiro, T.; Ogawa, T.; Sasahara, H. Temperature measurement of cutting tool and machined surface layer in milling of CFRP. International Journal of Machine Tools and Manufacture 2013, 70, 63-69. 
22. Kchit, N.; Lancon, P.; Bossis, G. Thermoresistance and giant magnetoresistance of magnetorheological elastomers. Journal of Physics D: Applied Physics 2009, 42(10), 105506.

23. Li, W.; Zhou, Y.; Tian, T.; Alici, G. Creep and recovery behaviors of magnetorheological elastomers. Frontiers of Mechanical Engineering in China 2010, 5(3), 341-346.

24. Qi, S.; Yu, M.; Fu, J.; Zhu, M. Stress relaxation behavior of magnetorheological elastomer: experimental and modeling study. Journal of Intelligent Material Systems and Structures 2018, 29(2), 205-213.

25. Yu, G.J.; Lin, X.G.; Guo, F. Modeling and Verification of Relaxation Behavior for Magnetorheological Elastomers with Applied Magnetic Field. In Key Engineering Materials 2017, 730, 527-532.

26. Xu, Y.; Liu, T.; Liao, G.J.; Lubineau, G. Magneto-dependentstress relaxation of magnetorheological gels. Smart Materials and Structures 2017. 26(11), 115005.

27. Meharthaj, H.; Srinivasan, S.M.; Arockiarajan, A. Creep behavior of magnetorheological gels. Mechanics of Advanced Materials and Structures 2020, 27(13), 1031-1039.

28. Puente-Córdova, J.G.; Reyes-Melo, M.E.; Palacios-Pineda, L.M.; Martínez-Perales, I.A.; MartínezRomero, O.; Elías-Zúñiga, A. Fabrication and Characterization of Isotropic and Anisotropic Magnetorheological Elastomers, Based on Silicone Rubber and Carbonyl Iron Microparticles. Polymers 2018, 10, 1343.

29. Carlson, J.; Jolly, M. MR fluid, foam and elastomer devices. Mechatronics 2000, 10(4-5), 429-594.

30. Bocian, M.; Kaleta, J.; Lewandowski, D.; Przybylski, M. Tunable Absorption System based on magnetorheological elastomers and Halbach array: design and testing. Journal of Magnetism and Magnetic Materials 2017, 435, 46-57.

31. Xu, X.; Hou, J. A stress relaxation model for the viscoelastic solids based on the steady-state creep equation. Mechanics of Time-Dependent Materials 2011, 15, 29-39. 